\title{
El mapa mental aumentado en puzle: expectativas de futuros docentes
}

\author{
Cristian Ariza Carrasco \\ Juan Manuel Muñoz González \\ Universidad de Córdoba. España. \\ m92arcac@uco.es \\ juan.manuel@uco.es
}

Recibido: 11/1/2021

Aceptado: 12/3/2021

Publicado: 23/9/2021

\section{Resumen}

El presente artículo tiene por objetivo mostrar las expectativas que los futuros docentes tienen sobre los beneficios de la técnica del mapa mental aumentado en puzle en los procesos de enseñanza-aprendizaje del alumnado. La muestra del estudio estuvo formada por 388 estudiantes de los grados de Educación Infantil y Primaria, así como del máster en Educación Inclusiva de la Universidad de Córdoba (España). Los datos fueron recogidos mediante un cuestionario creado ad hoc y conformado por una escala Likert (1-5) con doce ítems distribuidos entre tres dimensiones: «Desarrollo de las capacidades personales», "Desarrollo de las capacidades interpersonales» $\mathrm{y}$ "Desarrollo de las capacidades centradas en el aprendizaje». Los datos obtenidos se sometieron a análisis descriptivos, comparativa de medias, correlaciones y estudio de regresiones lineales. Los resultados muestran una valoración positiva de los futuros docentes ante las posibilidades de esta técnica a la hora de desarrollar las capacidades personales e interpersonales, así como las capacidades centradas en el aprendizaje de su futuro alumnado, por lo que la consideran un buen recurso a emplear en las aulas como educadores.

Palabras clave: mapa mental; realidad aumentada; aprendizaje cooperativo; formación inicial docente

\section{Resum. El mapa mental augmentat a puzle: expectatives de futurs docents}

El present article té per objectiu mostrar les expectatives que els futurs docents tenen sobre els beneficis de la tècnica del mapa mental augmentat a puzle en els processos d'ensenyament-aprenentatge de l'alumnat. La mostra de l'estudi va estar formada per 388 estudiants dels graus d'Educació Infantil i Primària, així com del màster en Educació Inclusiva de la Universitat de Còrdova (Espanya). Les dades van ser recollides mitjançant un qüestionari creat ad hoc i conformat per una escala Likert (1-5) amb dotze ítems distribuïts entre tres dimensions: desenvolupament de les capacitats personals, desenvolupament de les capacitats interpersonals i desenvolupament de les capacitats centrades en l'aprenentatge. Les dades obtingudes es van sotmetre a anàlisis descriptives, comparativa de mitjanes, correlacions i estudi de regressions lineals. Els resultats mostren una valoració positiva dels futurs docents davant les possibilitats d'aquesta tècnica a l'hora de desenvolupar les capacitats personals i interpersonals, així com les capacitats centrades en l'aprenentat- 
ge del seu futur alumnat, de manera que la consideren un bon recurs per emprar-lo a les aules com a educadors.

Paraules clau: mapa mental; realitat augmentada; aprenentatge cooperatiu; formació inicial docent

Abstract. The augmented mind map in puzzle: Expectations of future teachers

This article addresses the expectations of future teachers regarding the benefits of the augmented mind map in puzzle technique in the teaching-learning processes of students. The study sample comprised 388 students from the bachelor's degree programs in early childhood and primary education, as well as the Master in Inclusive Education at the University of Cordoba, Spain. The data were collected using a 5-point Likert scale questionnaire created ad hoc and composed of twelve items that were distributed in three dimensions: development of personal skills, development of interpersonal skills, and development of learning-focused skills. The data were subjected to descriptive analysis, comparative means, correlations, and the study of linear regressions. The results show that future teachers view the possibilities of this technique positively for developing the personal, interpersonal, and learning-focused skills of their students and consider it a good resource to use in the classroom as educators.

Keywords: mind map; augmented reality; cooperative learning; initial teaching training

\author{
Sumario \\ 1. Introducción 4. Discusión y conclusiones \\ 2. Método Referencias bibliográficas
}

3. Resultados

\title{
1. Introducción
}

Dado el papel de las TIC en la sociedad actual, es necesario que los futuros docentes reciban formación sobre el uso de metodologías que las integren y en las que el estudiante sea el protagonista de su propio proceso de enseñanzaaprendizaje.

Ante esta situación, se pueden encontrar en la literatura científica metodologías en las que el alumnado adquiere un papel protagonista, como es en el caso de los mapas mentales (Buzán, 1996); de técnicas de aprendizaje cooperativo como la técnica Jigsaw (Aronson et al., 1978), y en el uso de la realidad aumentada como recurso tecnológico con gran impacto en el sistema educativo actual (Cabero y García, 2016).

El mapa mental, creado por Buzán (1996), es una estructura gráfica que permite organizar las ideas sobre un tema en torno a un concepto principal, siguiendo una estructura radial con ramificaciones y empleando palabras clave, colores, formas y dibujos para representar los conceptos (Ontoria, Gómez y De Luque, 2003). 
Esta herramienta puede ser empleada tanto para el trabajo individual, en el que cada discente elabora su propio mapa mental del contenido abordado en el aula, como para el trabajo en grupos reducidos, en donde los estudiantes comparten sus mapas mentales y elaboran uno conjuntamente (Ontoria et al., 2003), fomentado así el desarrollo de habilidades sociales y la organización del trabajo (Guzmán, 2018; Muñoz-González, Marín-Díaz e Hidalgo-Ariza, 2020a; Muñoz-González, Vega-Gea e Hidalgo-Ariza, 2020b; Stokhof, De Vries, Bastiaens y Martens, 2019).

Por otro lado, este organizador gráfico ha sido empleado en los distintos niveles educativos para facilitar el aprendizaje y la comprensión de información, como también para guiar el pensamiento colectivo, obteniendo a su vez buenos resultados a la hora de enriquecer la creatividad y la motivación (Fuad, Zubaidah, Mahanal y Suarsini, 2017; Núñez Lira, Novoa Castillo, Majo Marrufo y Salvatierra Melgar, 2019; Stokhof et al., 2019).

En cuanto a la realidad aumentada, existen varias definiciones, pero todas coinciden en señalar que es una tecnología que, por medio de dispositivos electrónicos (ordenadores, tabletas, teléfonos inteligentes, etc.), permite combinar elementos virtuales con elementos del mundo real, al crear una superposición de capas donde se puede visualizar información en diferentes formatos, como imágenes, vídeos, audios y elementos en 3D (Cabero y García, 2016).

Ha sido aplicada en varios sectores, entre ellos el educativo, en donde han sido reportados los siguientes beneficios:

- Enriquecimiento del aprendizaje. La realidad aumentada es un buen medio para mejorar la comprensión y el aprendizaje, al facilitar la visualización y la interacción con la información, y promueve el desarrollo de habilidades cognitivas y motrices (Marín Díaz, Sampedro Requena y Muñoz González, 2017; Marín Díaz y Sampedro Requena, 2020; Martínez Pérez, Fernández Robles y Barroso Osuna, 2021).

- Componente motivacional. El uso de esta tecnología genera altos niveles de satisfacción e interés en el alumnado, dado que promueve la autonomía y la creatividad (Cabero Almenara, Vázquez-Cano, López Meneses y Jaén Martínez, 2020; López-Belmonte, Pozo-Sánchez y Fuentes-Cabrera, 2019; Marín Díaz et al., 2017; Moreno Fuentes y Pérez García, 2017; RodríguezGarcía, Hinojo-Lucena y Ágreda-Montoro, 2019).

- Fomento del trabajo en equipo. A la hora de trabajar en grupo, la realidad aumentada genera compromiso y colaboración, a la vez que ejerce una influencia positiva sobre la inclusión (Marín Díaz et al., 2017).

A su vez, tanto docentes como futuros maestros muestran una aceptación positiva hacia esta tecnología, dada su capacidad para generar un aprendizaje inmersivo y experimental, y su posibilidad de uso de forma colaborativa (Fernández Robles, 2018).

Por último, respecto a la técnica de aprendizaje cooperativo Jigsaw, que fue diseñada por Aronson en 1971 para resolver los conflictos raciales que surgieron 
en su aula (Mondéjar, Vargas y Meseguer, 2007), hay que destacar que constituye una metodología de trabajo en grupo que sigue las siguientes fases:

- Formación de grupos heterogéneos de trabajo de 4 a 6 miembros.

- División del tema a investigar por el alumnado en tantas partes como miembros tenga el grupo, para que cada uno reciba un fragmento diferente del tema.

- Los expertos en cada tema se reúnen en los llamados grupos de expertos para intercambiar información, debatir y redactar un informe.

- Finalizada la labor en el grupo de expertos, cada estudiante vuelve a su grupo de origen y expone su parte al resto de componentes.

Numerosos estudios desarrollados desde sus inicios han demostrado su efectividad de cara a los siguientes aspectos:

- Desarrollo del aprendizaje. Esta técnica favorece el aprendizaje autónomo, la comprensión, la organización y la adquisición de conocimientos (Dwiniasih y Nugraha, 2019; Mayorga Fernández y Madrid Vivar, 2012; Mondéjar et al., 2007; Sánchez Ambriz, 2014; Yoshida, 2018).

- Mejora de las habilidades sociales. La técnica Jigsaw, debido a su estructura de trabajo, permite fomentar las relaciones positivas, la solidaridad y la cooperación, mejorando así el clima de convivencia (Ghaith, 2018; Mayorga Fernández y Madrid Vivar, 2012; Sánchez Ambriz, 2014).

En definitiva, considerando los beneficios de estos tres elementos descritos, se decidió unificarlos en una nueva técnica denominada mapa mental aumentado en puzle, que ha sido evaluada positivamente en el ámbito universitario (Ariza Carrasco y Muñoz González, 2019), por lo que el estudio que se expone a continuación tiene la finalidad de exponer las expectativas que genera esta técnica en futuros docentes de cara a su posible uso como educadores.

\section{Método}

Con el propósito de alcanzar el objetivo anterior, se diseñó esta investigación bajo un enfoque descriptivo, empleando una encuesta transversal de naturaleza cuantitativa y siguiendo una estrategia de investigación deductiva y estructurada, y se establecieron los siguientes objetivos específicos:

- Exponer las percepciones de los futuros docentes sobre la contribución que el mapa mental aumentado en puzle puede realizar al desarrollo de las capacidades personales, las capacidades interpersonales y las capacidades centradas en el aprendizaje de su futuro alumnado.

- Comparar la diferencia de medias en las tres dimensiones del cuestionario respecto a las variables independientes: sexo, edad, titulación y uso de 
dispositivos electrónicos (PC, tableta, teléfono inteligente y consola de videojuegos).

- Verificar la existencia de correlaciones entre las dimensiones que componen el Cuestionario sobre expectativas MMAP.

- Analizar cómo influye la dimensión «Desarrollo de las capacidades personales» y la dimensión "Desarrollo de las capacidades interpersonales» sobre la dimensión «Desarrollo de las capacidades centradas en el aprendizaje».

\subsection{Muestra}

La muestra escogida para este estudio fue seleccionada mediante un muestreo no probabilístico (Otzen y Manterola, 2017), dado que participaron estudiantes a los que los docentes de esta investigación impartieron clase durante el curso académico 2017-2018.

La muestra total estuvo compuesta por 388 participantes que, tras el proceso de normalización, se redujo a 271 , entre los que el $74,9 \%$ eran mujeres y el 25,1\%, hombres, con la siguiente distribución: 106 eran del grado de Educación Infantil (el 39,1\%); 130, del grado de Educación Primaria (el 48\%), y 35, del máster en Educación Inclusiva (el 12,9\%). A continuación, se muestra una tabla con la distribución entre la edad y el sexo (tabla 1).

Tabla 1. Relación de distribución entre edad y sexo

\begin{tabular}{cccc}
\hline Edad & Mujeres & Hombres & Total \\
\hline 19 años & $66,3 \%$ & $33,8 \%$ & $29,6 \%$ \\
\hline 20 años & $70,6 \%$ & $29,4 \%$ & $12,6 \%$ \\
\hline 21 años & $60 \%$ & $40 \%$ & $11,1 \%$ \\
\hline 22 años & $82,4 \%$ & $17,6 \%$ & $12,5 \%$ \\
\hline 23 años & $90,9 \%$ & $9,1 \%$ & $12,2 \%$ \\
24 años & $77,8 \%$ & $22,2 \%$ & $6,6 \%$ \\
\hline 25 años & $93,3 \%$ & $6,7 \%$ & $5,5 \%$ \\
\hline 26 años & $80 \%$ & $20 \%$ & $1,8 \%$ \\
\hline Más de 26 años & $81,8 \%$ & $18,2 \%$ & $8,1 \%$ \\
\hline
\end{tabular}

Fuente: elaboración propia.

\subsection{Instrumento}

Para poder recopilar información del alumnado participante se utilizó el Cuestionario sobre expectativas $M M A P$, que fue creado ad hoc y cumplimentado en línea y de forma anónima. Está formado por preguntas cerradas y con una escala Likert de cinco opciones de respuesta, que van de «totalmente en desacuerdo» (1) a "totalmente de acuerdo» (5) en las dimensiones «Desarrollo de 
las capacidades personales», «Desarrollo de las capacidades interpersonales» y "Desarrollo de las capacidades centradas en el aprendizaje». A su vez, está constituida por doce ítems que se estructuran de la siguiente forma:

- Desarrollo de las capacidades personales. Dimensión compuesta por tres ítems que engloba aspectos relacionados con la motivación, el aprendizaje autónomo, la imaginación y la creatividad.

- Desarrollo de las capacidades interpersonales. Abarca cinco ítems que recopilan información sobre la capacidad para dialogar y argumentar, el desarrollo de debates, la confianza a la hora de compartir ideas y el clima de convivencia generado.

- Desarrollo de las capacidades centradas en el aprendizaje. Esta dimensión se compone de cuatro ítems relacionados con el aprendizaje, como la capacidad para organizar, comprender y recordar la información, así como la asimilación de conceptos.

También incluye una serie de variables independientes de ámbito académico (titulación), sociodemográfico (edad, sexo) y sobre el uso de dispositivos electrónicos (ordenadores, tabletas digitales, teléfonos inteligentes y consola de videojuegos).

En lo referente a la validación y a la fiabilidad del instrumento, se llevaron a cabo varios análisis estadísticos. En primer lugar, se utilizó un análisis factorial exploratorio (AFE), empleando las matrices de correlación de Pearson, junto con el método para determinar el número de factores «Implementación óptima del análisis paralelo» (Timmerman y Lorenzo-Seva, 2011) y el procedimiento "cuadrados no ponderados robusto» con criterio de rotación Promin (Lorenzo-Seva, 2000), empleando los programas estadísticos SPSS 23 y Factor Analysis (10.8.04), que permitieron ver los criterios sobre su viabilidad: determinante de la matriz correlaciones con valor 0,$000 ; \mathrm{KMO}=0,95$; prueba de esfericidad de Barlett con $p=0,000$ y raíz del residuo cuadrático promedio: RMSR = 0,019; donde los factores extraídos explicaron un 80,88\% de la varianza. La matriz obtenida de los componentes rotados mostró que el peso factorial de cada uno de los ítems (tabla 2) presenta cargas superiores a 0,3, guardando coherencia con las dimensiones asignadas para cada uno.

En segundo lugar, se realizó un análisis factorial confirmatorio (AFC), considerándose la prueba $\chi 2 /$ grados de libertad, el índice de bondad de ajuste comparativo (CFI), el índice de ajuste incremental (IFI), el índice de ajuste normado (NFI), el índice de Tuker-Lewis (TLI), la raíz del residuo cuadrático promedio de aproximación (RMSEA) y el índice de validación cruzada esperada (ECVI), en los que se obtuvo los siguientes valores: $\mathrm{X}^{2}=59,49 ; d f=45$; $p=0,07 ; \mathrm{X}^{2} / d f=1,32 ; \mathrm{CFI}=0,99 ; \mathrm{IFI}=0,99$; NFI $=0,97$; TLI $=0,99$; RMSEA $=0,03$, y ECVI $=0,55$.

Por último, se examinó la fiabilidad del instrumento por medio del coeficiente Alpha de Cronbach, mostrando la existencia de una fiabilidad alta, tanto del instrumento completo $(\alpha=0,94)$ como de cada una de sus dimensiones 
Tabla 2. Matriz de factores rotados del análisis factorial exploratorio

\begin{tabular}{|c|c|c|c|}
\hline Variable & F1 & F2 & F3 \\
\hline V 1 & 0,548 & & \\
\hline V 2 & 0,484 & & \\
\hline V 3 & 0,678 & & \\
\hline V 4 & & 0,961 & \\
\hline V 5 & & 1,044 & \\
\hline V 6 & & 1,105 & \\
\hline$\vee 7$ & & 0,611 & \\
\hline V 8 & & 0,624 & \\
\hline V 9 & & & 0,557 \\
\hline V 10 & & & 0,823 \\
\hline V 11 & & & 1,040 \\
\hline V 12 & & & 1,069 \\
\hline
\end{tabular}

Fuente: elaboración propia.

( $\alpha=0,78$ en el factor $1 ; \alpha=0,90$ en el factor 2 , y $\alpha=0,90$ en el factor 3 ) (Thorndike, 1997).

\subsection{Procedimiento de recogida y análisis de datos}

La dinámica de aprendizaje llevada a cabo en el presente estudio se desarrolló a lo largo de siete sesiones de clase y se estructuró en una serie de fases que se detallan a continuación:

- División del tema. Se escogió un tema y se dividió en varias partes.

- Creación de los grupos base de trabajo. En esta fase se formaron los grupos de trabajo cooperativo, compuestos por un número de miembros igual al número de partes en las que se dividió el tema, para asignar una parte distinta a cada estudiante.

- Investigación y elaboración del mapa mental. Los discentes emplearon el material que se les proporcionó para elaborar un mapa mental.

- Reunión del grupo de expertos. Estos grupos fueron formados por estudiantes con el mismo tema investigado para que, empleando el mapa mental previamente diseñado, compartieran la información recabada con el resto de los miembros del grupo. Al mismo tiempo tuvieron que actualizar el contenido de sus mapas mentales y redactar un resumen con el conjunto de la información entre todos los componentes del grupo.

- Elaboración del mapa mental aumentado grupal. Finalizado el paso anterior, cada estudiante regresó a su grupo base de trabajo para explicar su parte al resto de miembros del equipo, empleando el mapa mental elabo- 
rado a mano y, entre todos, elaboraron con el programa MindManager un mapa mental aumentado con todas las partes del tema, y al que le incorporaron elementos de realidad aumentada por medio del software Augment.

Una vez concluido el trabajo, cumplimentaron en línea y de forma anónima el Cuestionario sobre expectativas MMAP, y se realizaron los siguientes análisis:

- En primer lugar, se llevó a cabo un análisis descriptivo de las doce variables incluidas en el cuestionario por medio de medidas de tendencia central (media) y de dispersión (desviación típica).

- En segundo lugar, se empleó un análisis descriptivo de las tres dimensiones del cuestionario.

- En tercer lugar, se comprobó la existencia de diferencias significativas entre cada una de las dimensiones del cuestionario con las variables independientes (sexo, edad, titulación y uso de dispositivos electrónicos por parte del alumnado) por medio de la $t$ de Student y ANOVA, utilizando el paquete estadístico SPSS 23.

- En cuarto lugar, emplearon correlaciones bivariadas para comprobar la existencia de relaciones entre las dimensiones que componen el cuestionario.

- En último lugar, se estudió la influencia que ejerce la dimensión 1, «Desarrollo de las capacidades personales», y la dimensión 2, «Desarrollo de las capacidades interpersonales», sobre la dimensión 3, «Desarrollo de las capacidades centradas en el aprendizaje», por medio de regresiones lineales.

\section{Resultados}

Los resultados de este estudio comienzan con el análisis descriptivo (media y desviación típica) de los doce ítems del Cuestionario sobre expectativas MMAP (tabla 3).

Tabla 3. Distribución de frecuencias de los ítems del Cuestionario sobre expectativas MMAP

\begin{tabular}{llcc}
\hline Dimensión & Ítems & M & DT \\
\hline $\begin{array}{l}\text { Factor 1. Desarrollo } \\
\text { de las capacidades }\end{array}$ & $\begin{array}{l}\text { 1. El alumnado puede encontrar esta dinámica de trabajo } \\
\text { personales }\end{array}$ & 3,99 & 0,86 \\
& $\begin{array}{l}\text { 2. La dinámicara del mapa mental aumentado en puzle per- } \\
\text { mitirá al alumnado aprender de forma autónoma. }\end{array}$ & 3,96 & 0,76 \\
& $\begin{array}{l}\text { 3. La dinámica del mapa mental aumentado en puzle con- } \\
\text { tribuirá al desarrollo de la imaginación y la creatividad } \\
\text { del alumnado. }\end{array}$ & 4,07 & 0,78 \\
&
\end{tabular}


Factor 2. Desarrollo de las capacidades interpersonales

Factor 3. Desarrollo de las capacidades centradas en el aprendizaje
4. La dinámica del mapa mental aumentado en puzle ayudará al alumnado a mejorar su capacidad para dialogar.

5. La dinámica del mapa mental aumentado en puzle ayudará al alumnado a desarrollar su capacidad de argumentación.

6. La dinámica del mapa mental aumentado en puzle facili- $\quad 3,91 \quad 0,82$ tará el desarrollo de debates entre el alumnado.

7. La dinámica del mapa mental aumentado en puzle ayu- $\begin{array}{lll}3,98 & 0,78\end{array}$ dará al alumnado a desarrollar su confianza a la hora de compartir ideas con los compañeros.

8. La dinámica del mapa mental aumentado en puzle con- $\quad 3,91 \quad 0,77$ tribuirá al desarrollo de un clima de convivencia positivo en el aula.

9. La dinámica del mapa mental aumentado en puzle ayu- $\quad 4,18 \quad 0,73$ dará al alumnado a organizar la información.

10. La dinámica del mapa mental aumentado en puzle favo- $\quad 4,03 \quad 0,83$ recerá una mejor comprensión de los contenidos.

11. La dinámica del mapa mental aumentado en puzle posi- $\quad \begin{array}{lll}4,03 & 0,81\end{array}$ bilitará una mejor asimilación de contenidos.

12. La dinámica del mapa mental aumentado en puzle $\quad 4,07 \quad 0,74$ mejorará la capacidad del alumnado para recordar la información.

Fuente: elaboración propia.

A continuación, los resultados se han estructurado en función de los objetivos específicos de este estudio.

\subsection{Contribuciones del mapa mental aumentado en puzle en función de las capacidades del alumnado}

Los análisis descriptivos realizados con cada una de las dimensiones que abarca el instrumento diseñado arrojaron los siguientes resultados (tabla 4).

En la dimensión «Desarrollo de capacidades personales», conformada por tres ítems que midieron la motivación, el aprendizaje autónomo, la imaginación y la creatividad, los análisis indicaron una mediana de 4 y una media de 4 , por lo que el alumnado muestra una actitud de estar parcialmente de acuer-

Tabla 4. Estadísticos descriptivos por dimensiones

\begin{tabular}{lccc}
\hline & $\begin{array}{c}\text { Desarrollo de } \\
\text { las capacidades } \\
\text { personales }\end{array}$ & $\begin{array}{c}\text { Desarrollo de } \\
\text { las capacidades } \\
\text { interpersonales }\end{array}$ & $\begin{array}{c}\text { Desarrollo de las } \\
\text { capacidades centradas } \\
\text { en el aprendizaje }\end{array}$ \\
\hline Media & 4 & 3,94 & 4,07 \\
\hline Mediana & 4 & 4 & 4 \\
\hline $\begin{array}{l}\text { Desviación } \\
\text { estándar }\end{array}$ & 0,66850 & 0,67452 & 0,68765 \\
Mínimo & 1,67 & 2,00 & 1,50 \\
\hline Máximo & 5,00 & 5,00 & 5,00 \\
\hline
\end{tabular}

Fuente: elaboración propia. 
do sobre la utilidad del mapa mental aumentado en puzle a la hora de desarrollar estas capacidades de sus futuros discentes; siendo la opción 4 de respuestas la que presenta mayor porcentaje (un 48,1\%), acumulando un porcentaje entre 4 y 5 de un 76,26\%, y con una opción mínima de 1,67, en la que solo se encontró a una persona que se posicionó totalmente en desacuerdo con los ítems incluidos en la dimensión.

En lo referente a la dimensión «Desarrollo de las capacidades interpersonales», compuesta por un total de 5 ítems que abordan elementos relacionados con la capacidad para dialogar y argumentar, desarrollar debates y generar confianza a la hora de compartir información y crear un clima de convivencia positivo, los resultados reflejan una mediana de 4 y una media de 3,94, mostrando así una actitud de indiferencia, aunque muy próxima a "parcialmente de acuerdo". Al igual que en el caso anterior, la opción 4 de respuestas es la que mayor porcentaje acumula (un 48,26\%), siendo el porcentaje acumulado entre 4 y 5 de $72,78 \%$, pero el mínimo en las opciones de respuestas fue 2 .

Por último, en la dimensión «Desarrollo de las capacidades centradas en el aprendizaje», que recopila en sus 4 ítems información sobre las capacidades de aprendizaje relacionadas con la organización, la comprensión, el recuerdo y la asimilación de contenidos, los resultados obtenidos muestran una mediana de 4 y una media de 4,07, mostrando los estudiantes una postura de «parcialmente de acuerdo". A su vez, tal y como ha sucedido en las dimensiones anteriores, la opción de respuesta más repetida es la 4 , con un porcentaje de un $48,32 \%$, y acumulando entre las opciones 4 y 5 un 79,52\%, siendo el valor menor obtenido de 1,5 , por lo que hay una persona que se posicionó totalmente en desacuerdo con los ítems evaluados en la dimensión.

\subsection{Estudio inferencial}

La comparativa de medias de las dimensiones del cuestionario con las variables independientes sexo, edad, titulación y uso de dispositivos electrónicos (ordenador, tableta, teléfono inteligente y consola de videojuegos) arrojaron los siguientes resultados:

Para las variables sexo y uso de dispositivos electrónicos se empleó la $t$ de Student para muestras independientes, que no evidenció diferencias estadísticamente significativas en ninguna de las tres dimensiones.

Respecto a las variables independientes titulación y edad, se utilizó el análisis de varianza (ANOVA), no obteniendo diferencias estadísticamente significativas.

\subsection{Análisis correlacional}

En este apartado se ha realizado un estudio correlacional, por medio de la correlación de Pearson, entre las tres dimensiones del cuestionario. Los resultados obtenidos son los siguientes (tabla 5). 
Tabla 5. Resultados de las correlaciones bivariadas de los ítems de las tres dimensiones del cuestionario

\begin{tabular}{|c|c|c|c|c|}
\hline & & $\begin{array}{c}\text { Desarrollo de } \\
\text { las capacidades } \\
\text { personales }\end{array}$ & $\begin{array}{c}\text { Desarrollo de } \\
\text { las capacidades } \\
\text { interpersonales }\end{array}$ & $\begin{array}{c}\text { Desarrollo de } \\
\text { las capacidades } \\
\text { centradas en el } \\
\text { aprendizaje } \\
\end{array}$ \\
\hline \multirow{3}{*}{$\begin{array}{l}\text { Desarrollo de } \\
\text { las capacidades } \\
\text { personales }\end{array}$} & $N$ & 271 & 271 & 271 \\
\hline & $\begin{array}{l}\text { Correlación } \\
\text { de Pearson }\end{array}$ & 1 & $0,73^{* *}$ & $0,72^{* *}$ \\
\hline & Sig. (bilateral) & & 0,00 & 0,00 \\
\hline \multirow{3}{*}{$\begin{array}{l}\text { Desarrollo de } \\
\text { las capacidades } \\
\text { interpersonales }\end{array}$} & $N$ & 271 & 271 & 271 \\
\hline & $\begin{array}{l}\text { Correlación } \\
\text { de Pearson }\end{array}$ & $0,73^{* *}$ & 1 & $0,79^{* *}$ \\
\hline & Sig. (bilateral) & 0,00 & & 0,00 \\
\hline \multirow{3}{*}{$\begin{array}{l}\text { Desarrollo de } \\
\text { las capacidades } \\
\text { centradas en el } \\
\text { aprendizaje }\end{array}$} & $N$ & 271 & 271 & 271 \\
\hline & $\begin{array}{l}\text { Correlación } \\
\text { de Pearson }\end{array}$ & $0,72^{* *}$ & $0,79^{* *}$ & 1 \\
\hline & Sig. (bilateral) & 0,00 & 0,00 & \\
\hline
\end{tabular}

** La correlación es significativa en el nivel 0,01 (bilateral).

Fuente: elaboración propia.

Observando los datos, se aprecia que existe una correlación elevada (Mateo, 2004; Pérez, García, Gil y Galán, 2009) entre la dimensión 1, «Desarrollo de las capacidades personales», con la dimensión 2, «Desarrollo de las capacidades interpersonales», y la dimensión 3, "Desarrollo de las capacidades centradas en el aprendizaje» $(R=0,73$ y $p=0,00 ; R=0,72$ y $p=0,00)$.

Por otra parte, también existe relación entre la dimensión 2 , «Desarrollo de las capacidades interpersonales», y la dimensión 3, "Desarrollo de las capacidades centradas en el aprendizaje» $(R=0,79$ y $p=0,00)$, presentando igualmente una correlación alta como en la situación anterior.

\subsection{Modelos explicativos del desarrollo de las capacidades centradas en el aprendizaje}

Con el objetivo de explicar la dimensión «Desarrollo de las capacidades centradas en el aprendizaje» a través de las medidas de las dimensiones "Desarrollo de las capacidades personales" $\mathrm{y}$ "Desarrollo de las capacidades interpersonales», se han empleado regresiones lineales múltiples (Pardo y Ruiz, 2002) utilizando el método por pasos para observar la conexión entre la variable predictora o explicativa y la variable criterio (tabla 6). 
Tabla 6. Coeficiente de la recta de regresión para la dimensión «Desarrollo de las capacidades centradas en el aprendizaje»

\begin{tabular}{lcccccccc}
\hline \multicolumn{7}{c}{ Coeficientes $^{\mathbf{a}}$} \\
\hline \multicolumn{1}{c}{ Modelo } & $\begin{array}{c}\text { Coeficientes no } \\
\text { estandarizados }\end{array}$ & $\begin{array}{c}\text { Coeficientes } \\
\text { estandarizados }\end{array}$ & $\boldsymbol{t}$ & Sig. & \multicolumn{2}{c}{$\begin{array}{c}\text { Estadísticas de } \\
\text { colinealidad }\end{array}$} \\
\cline { 2 - 9 } & B & Error estándar & Beta & & \multicolumn{3}{c}{ Tolerancia } & VIF \\
\hline 1 (Constante) & 0,53 & 0,15 & & 3,39 & 0,001 & & \\
$\begin{array}{l}\text { Desarrollo de las } \\
\text { capacidades per- } \\
\text { sonales }\end{array}$ & 0,32 & 0,05 & 0,31 & 6,15 & 0,000 & 0,47 & 2,14 \\
$\begin{array}{l}\text { Desarrollo de } \\
\text { las capacidades } \\
\text { interpersonales }\end{array}$ & 0,57 & 0,05 & 0,56 & 10,96 & 0,000 & 0,47 & 2,14 \\
\hline
\end{tabular}

Fuente: elaboración propia.

En los resultados se aprecia que la dimensión «Desarrollo de las capacidades centradas en el aprendizaje» es predicha por las dimensiones "Desarrollo de las capacidades personales" y "Desarrollo de las capacidades interpersonales», ya que $\beta=0,32 ; t(32)=6,15, p<0,001, \mathrm{y} \beta=0,57 ; t(57)=10,96$, $p<0,001$, por lo que es estadísticamente significativa, aceptando así la hipótesis de relación lineal entre las dos dimensiones con la dimensión «Desarrollo de las capacidades centradas en el aprendizaje». Por último, hay que destacar que el valor de $\mathrm{R}^{2}$ ajustado fue de 0,67 , lo que indica que el $67 \%$ de la variabilidad del desarrollo de las capacidades centradas en el aprendizaje es explicada por el desarrollo de las capacidades personales e interpersonales.

\section{Discusión y conclusiones}

En este estudio, como se ha podido comprobar a raíz de los resultados obtenidos, se aprecia que los futuros docentes que experimentaron con la técnica del mapa mental aumentado en puzle la percibían como un recurso a tener en cuenta en su futuro trabajo como educadores. Este hecho se ha visto reflejado en la valoración realizada en cada una de las dimensiones analizadas en esta investigación.

En la primera dimensión, centrada en el desarrollo de las capacidades personales, y en donde se midió la motivación, el aprendizaje autónomo y el desarrollo de la imaginación y la creatividad; el alumnado mostró una valoración positiva en cuanto al potencial de la técnica para desarrollar estos aspectos en su futuro alumnado, que no se vio influenciada por variables relacionadas con el sexo, la edad, la titulación o el uso de dispositivos electrónicos. Estos resultados coinciden con estudios en los que se ha comprobado que durante el trabajo con la técnica Jigsaw en las diferentes etapas educativas, tanto los docentes (Ghaith, 2018; Sánchez Ambriz, 2014) como el alumnado han destacado que ayuda a promover una mayor motivación, autonomía y diversión durante el trabajo en el aula (Mayorga Fernández y Madrid Vivar, 2012; Nur- 
malia, Halim y Syahrun, 2020; Ural, Ercan y Gençoğlan, 2017), al igual que sucede durante el trabajo con mapas mentales (Muñoz González, Ariza Carrasco y Sampedro Requena, 2015; Muñoz-González et al., 2020b; Núñez Lira et al., 2019) o con la realidad aumentada (Cabero-Almenara, Vázquez-Cano y López Meneses, 2018; Fernández Robles, 2018; Hung, Chen y Huang, 2017; Moreno Fuentes y Pérez García, 2017; Rodríguez-García et al., 2019), los cuales poseen también un componente motivacional, siendo herramientas que fomentan la autonomía y la creatividad. A su vez, contrasta con investigaciones en donde ha sido más valorada la realidad aumentada por estudiantes de menor edad (Marín Díaz et al., 2017).

Por otro lado, en la dimensión relacionada con el desarrollo de las capacidades interpersonales, pese a no llegar al mismo nivel de valoración que la anterior, las respuestas se aproximaron bastante a una actitud favorable con los elementos evaluados (capacidad de argumentación y diálogo, desarrollo de debates, mejora de la autoconfianza al compartir ideas y desarrollar un clima de convivencia positivo). Este hecho está en consonancia con la potencialidad del mapa mental (Muñoz González et al., 2015; Muñoz-González et al., 2020b; Stokhof et al., 2019), la realidad aumentada (Marín Díaz et al., 2017) y la técnica Jigsaw (Ghaith, 2018; Mayorga Fernández y Madrid Vivar, 2012; Sánchez Ambriz, 2014) para fomentar el respeto y el diálogo entre el alumnado de las diferentes etapas educativas y para generar un buen clima de convivencia. Así mismo, variables como el género, la edad, la titulación y el uso de dispositivos electrónicos no han influido en su evaluación (Ariza Carrasco y Muñoz González, 2019), a diferencia de estudios en donde el género ha sido un factor diferenciador al medir la vertiente cooperativa del mapa mental (Muñoz-González et al., 2020b), así como la edad y el género en la realidad aumentada (Marín y Sampedro-Requena, 2020).

Tanto la dimensión «Desarrollo de las capacidades personales» como la dimensión «Desarrollo de las capacidades interpersonales» mostraron ser factores predictivos de la dimensión «Desarrollo de las capacidades centradas en el aprendizaje», con un alto grado de interrelación entre ellas (Muñoz-González et al., 2020b).

En lo que respecta a esta última dimensión, los estudiantes se mostraron parcialmente de acuerdo con la eficacia de la técnica para mejorar en su futuro alumnado aspectos vinculados al desarrollo de las capacidades centradas en el aprendizaje, como la comprensión, la organización, el recuerdo y la asimilación de información, sin influir en dicha apreciación las variables independientes tenidas en cuenta en el cuestionario, en contraposición a estudios en donde las mujeres han evaluado con mayor puntuación la capacidad de aprendizaje que genera el mapa mental (Muñoz-González et al., 2020b). Estos resultados coinciden con aportaciones de otros investigadores en las que se cita al mapa mental (Fuad et al., 2017; Guzmán, 2018; Muñoz González et al., 2015; Muñoz-González et al., 2020b), a la realidad aumentada (Cabero-Almenara et al., 2018; Fernández Robles, 2018; Marín Díaz y Muñoz Asencio, 2018; Marín y Sampedro-Requena, 2020) y al aprendizaje cooperativo (Dwiniasih 
y Nugraha, 2019; Herman, Sibarani y Pardede, 2020; Navarro, González, López y Contreras, 2019; Ural et al., 2017; Yoshida, 2018) como herramientas que son tenidas en cuenta por futuros docentes parar aplicar en el aula y que ayudan en los procesos de aprendizaje del alumnado de los distintos niveles educativos.

En definitiva, los futuros docentes consideran que la aplicación de la técnica del mapa mental aumentado en puzle en las aulas puede ayudarles como educadores a desarrollar las capacidades personales e interpersonales, además de aquellas centradas en el aprendizaje de su futuro alumnado.

Pese a los resultados alcanzados, se desconoce la valoración que los docentes en activo harían a esta técnica, ya que el estudio está focalizado en las expectativas que ha generado en futuros docentes, por lo que para venideras líneas de investigación sería idóneo conocer la opinión que tienen los educadores de los diferentes niveles académicos sobre su utilidad para la enseñanza.

\section{Referencias bibliográficas}

Ariza Carrasco, C. y Muñoz González, J.M. (2019). Valoración de la técnica del mapa mental aumentado en puzle en educación superior. REOP-Revista Española de Orientación y Psicopedagogía, 30(3), 64-83. <https://doi.org/10.5944/reop.vol.30.num.3.2019.26273>

Aronson, E.; Stephan, C.; Sikes, J.; Blaney, N. y Snapp, M. (1978). The Jigsaw Classroom. Beverly Hills, California: Sage Publications.

Buzán, T. (1996). The Mind Map Book: How to Use Radiant Thinking to Maximize Your Brain's untapped potential. Nueva York (USA): Plume.

Cabero-Almenara, J.; VÁzquez-Cano, E. y López-Meneses, E. (2018). Uso de la realidad aumentada como recurso didáctico en la enseñanza universitaria. Formacion Universitaria, 11(1), 25-34.

Cabero Almenara, J.; VÁzquez-Cano, E.; López Meneses, E. y Jaén Martínez, A. (2020). Posibilidades formativas de la tecnología aumentada: Un estudio diacrónico en escenarios universitarios. Revista Complutense de Educación, 31(2), 141-152. $<$ https://doi.org/10.5209/rced.61934>

Cabero, J. y García, F. (2016). Realidad aumentada, tecnología para la formación. Madrid: Síntesis.

Dwiniasih, D. y Nugraha, A.F. (2019). Enhancing Students' Reading Comprehension Through Jigsaw. Academic Journal Perspective: Education, Language, and Literature, $7(1), 46-50$. <http://dx.doi.org/10.33603/perspective.v7i1.1909>

Fernández Robles, B. (2018). La utilización de objetos de aprendizaje de realidad aumentada en la enseñanza universitaria de Educación Primaria. IJERI: International Journal of Educational Research and Innovation, 9, 90-104. Recuperado de $<$ https://www.upo.es/revistas/index.php/IJERI/article/view/2599>.

Fuad, N.M.; Zubaidah, S.; Mahanal, S. y Suarsini, E. (2017). Improving junior high schools' critical thinking skills based on test three different models of learning. International Journal of Instruction, 10(1), 101-116.

Gнaith, G.M. (2018). Teacher perceptions of the challenges of implementing concrete and conceptual cooperative learning. Issues in Educational Research, 28(2), 385-404. Recuperado de <http://www.iier.org.au/iier28/ghaith.pdf>. 
GuZMán, L.R. (2018). Los mapas mentales como estrategia de comprensión lectora y recurso tecnológico de apoyo. Leiria: Escola Superior de Educação e Ciências Sociais.

Herman; Sibarani, J.K. y Pardede, H. (2020). The Effect of Jigsaw Technique in Reading Comprehension on Recount Text. Cetta: Jurnal Ilmu Pendidikan, 3(1), 84-102. $<$ https://doi.org/10.37329/cetta.v3i1.413>

Hung, Y.-H.; Chen, C.-H. y Huang, S.-W. (2017). Applying augmented reality to enhance learning: A study of different teaching materials. Journal of Computer Assisted Learning, 33, 252-266. <https://doi.org/10.1111/jcal.12173>

López-Belmonte, J.; Pozo-Sánchez, S. y Fuentes-Cabrera, A. (2019). Technopedagogical resources to support teaching: Augmented reality as a dynamic tool for the substitute teacher. IJERI: International Journal of Educational Research and Innovation, 12, 122-136.

<https://doi.org/10.46661/ijeri.3653>

Lorenzo-Seva, U. (2000). The weighted oblimin rotation. Psychometrika, 65(3), 301-318. <https://doi.org/10.1007/BF02296148>

Marín Díaz, V. y Muñoz Asencio, V.P. (2018). Trabajar el cuerpo humano con realidad aumentada en educación infantil. Revista Tecnología, Ciencia y Educación, 9, 148-158. Recuperado de <https://tecnologia-ciencia-educacion.com/index.php/ TCE/article/view/177/141>.

Marín Díaz, V.; Sampedro Requena, B.E. y Muñoz González, J.M. (2017). La Realidad Aumentada en el aula de Educación Primaria. Revista Observatório, 3(5), 634-668. <https://doi.org/10.20873/uft.2447-4266.2017v3n5p634>

Marín, V. y SAmpedro-RequenA, B.E. (2020). La realidad aumentada en Educación Primaria desde la visión de los estudiantes. Alteridad, 15(1), 61-73. <https://doi.org/10.17163/alt.v15n1.2020.05>

Martínez Pérez, S.; Fernández Robles, B. y Barroso Osuna, J. (2021). La realidad aumentada como recurso para la formación en la educación superior. Campus Virtuales, 10(1), 9-19.

Mateo, J. (2004). La investigación 'ex post-facto'. En R. BisQuerra (coord.). Metodología de investigación educativa (pp. 196-230). Madrid: La Muralla.

Mayorga Fernández, M.J. y Madrid Vivar, D. (2012). La técnica del puzle como estrategia de aprendizaje cooperativo para la mejora del rendimiento académico. Publicaciones de la Facultad de Educación y Humanidades del Campus de Melilla, 42, 89-106.

MondéJar, J.; Vargas, M. y Meseguer, M.L. (2007). Aprendizaje cooperativo en entornos virtuales: El método Jigsaw en asignaturas de estadística. Documentos de Trabajo: Seminario Permanente de Ciencias Sociales, 3, 1-18.

Moreno Fuentes, E. y Pérez García, Á. (2017). La realidad aumentada como recurso didáctico para los futuros maestros. Revista Cientifica Electrónica de Educación y Comunicación en la Sociedad del Conocimiento, 1(17), 42-59.

Muñoz González, J.M.; Ariza Carrasco, C. y Sampedro Requena, B.E. (2015). La aplicación de los mapas mentales en educación primaria. International Journal of Educational Research and Innovation, 4, 70-89. Recuperado de <https://www. upo.es/revistas/index.php/IJERI/article/view/1459>.

Muñoz-González, J.-M.; Marín-Díaz, V. e Hidalgo-Ariza, M.-D. (2020a). Validación de una escala de medida del mapa mental como estrategia de aprendizaje en la formación inicial docente. Estudios sobre Educación, 38, 79-100.

<https://doi.org/10.15581/004.38.79-100> 
Muñoz-González, J.M.; Vega-Gea, E.M. e Hidalgo-Ariza, M.A. (2020b). El aprendizaje del mapa mental grupal mediante las TIC en Educación Superior. Educação \& Sociedade, 41. $<$ https://dx.doi.org/10.1590/es.219656>

Navarro, I.; González, C.; López, B. y Contreras, A. (2019). Aprendizaje cooperativo basado en proyectos y entornos virtuales para la formación de futuros maestros. Educar, 55(2), 519-541. $<$ https://doi.org/10.5565/rev/educar.935>

Núñez Lira, L.A.; Novoa Castillo, P.F.; Majo Marrufo, H.R. y Salvatierra MelGAR, A. (2019). Los mapas mentales como estrategia en el desarrollo de la inteligencia exitosa en estudiantes de secundaria. Propósitos y Representaciones, 7(1), 59-82. <https://dx.doi.org/10.20511/pyr2019.v7n1.263>

Nurmalia; Halim, A. y Syahrun, N. (2020). Application of jigsaw type cooperative learning to improve student creative thinking skills. Journal of Physics: Conference Series, 1460(1), 012142. <https://doi.org/10.1088/1742-6596/1460/1/012142>

Ontoria, A.; Gómez, J.P. y Luque, A. de (2003). Aprender con Mapas Mentales. Madrid: Narcea.

Otzen, T. y Manterola, C. (2017). Técnicas de muestreo sobre una población a estudio. International Journal of Morphology, 35(1), 227-232. <https://dx.doi.org/10.4067/S0717-95022017000100037>

Pardo, A. y Ruiz, M. (2002). SPSS 11: Guía para el análisis de datos. Madrid: McGraw-Hill.

Pérez, R.; García, J.L.; Gil, J.A. y Galán, A. (2009). Estadistica aplicada a la Educación. Madrid: Pearson Prentice Hall.

Rodríguez-García, A.M.; Hinojo-Lucena, F.J. y Ágreda-Montoro, M. (2019). Diseño e implementación de una experiencia para trabajar la interculturalidad en Educación Infantil a través de realidad aumentada y códigos QR. Educar, 55(1), 59-77. <https://doi.org/10.5565/rev/educar.966>

Sánchez Ambriz, M.L. (2014). Diseño y producción de cursos MOOC como estrategia de aprendizaje cooperativo en un ambiente de educación a distancia. Didáctica, Innovación y Multimedia: DIM, 28, 1-12.

Stokhof, H.; Vries, B. de; Bastiaens, T. y Martens, R. (2019). Mind Map Our Way into Effective Student Questioning: A Principle-Based Scenario. Research in Science Education, 49(2), 347-369. <https://doi.org/10.1007/s11165-017-9625-3>

Thorndike, R.M. (1997). Measurement and evaluation in psychology and education. 6. ${ }^{a}$ ed. Nueva York: McMillan.

Timmerman, M.E. y Lorenzo-Seva, U. (2011). Dimensionality assessment of ordered polytomous items with parallel analysis. Psychological Methods, 16(2), 209-220. <https://doi.org/10.1037/a0023353>

Ural, E.; Ercan, O. y GençOĞLan, D.M. (2017). The effect of jigsaw technique on 6th graders' learning of force and motion unit and their science attitudes and motivation. Asia-Pacific Forum on Science Learning and Teaching, 18(1), 1-21.

Yoshida, M. (2018). Communication Jigsaw: A Teaching Method that Promotes Scholarly Communication. International Journal of Emerging Technologies in Learning (iJET), 13(10), 208-224.

<http://dx.doi.org/10.3991/ijet.v13i10.8850> 